University of Nebraska - Lincoln

DigitalCommons@University of Nebraska - Lincoln

2011

\title{
Valproic acid inhibits neurosphere formation by adult subventricular cells by a lithium-sensitive mechanism
}

\author{
Q. Zhou \\ Uniformed Services University of the Health Sciences \\ C. L. Dalgard \\ Uniformed Services University of the Health Sciences \\ C. Wynder \\ University of Western Ontario \\ M. L. Doughty \\ Uniformed Services University of the Health Sciences, mdoughty@usuhs.mil
}

Follow this and additional works at: https://digitalcommons.unl.edu/usuhs

Part of the Medicine and Health Sciences Commons

Zhou, Q.; Dalgard, C. L.; Wynder, C.; and Doughty, M. L., "Valproic acid inhibits neurosphere formation by adult subventricular cells by a lithium-sensitive mechanism" (2011). Uniformed Services University of the Health Sciences. 78.

https://digitalcommons.unl.edu/usuhs/78

This Article is brought to you for free and open access by the U.S. Department of Defense at DigitalCommons@University of Nebraska - Lincoln. It has been accepted for inclusion in Uniformed Services University of the Health Sciences by an authorized administrator of DigitalCommons@University of Nebraska Lincoln. 


\title{
Valproic acid inhibits neurosphere formation by adult subventricular cells by a lithium-sensitive mechanism
}

\author{
Q. Zhou a , C.L. Dalgard ${ }^{\mathrm{a}, \mathrm{b}}$, C. Wynder ${ }^{\mathrm{c}}$, M.L. Doughty ${ }^{\mathrm{a}, \mathrm{b}, *}$

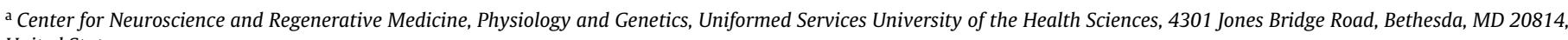 \\ United States \\ ${ }^{\mathrm{b}}$ Department of Anatomy, Physiology and Genetics, Uniformed Services University of the Health Sciences, 4301 Jones Bridge Road, Bethesda, MD 20814, United States \\ ' Department of Biochemistry, Schulich School of Medicine, University of Western Ontario, 112 SDRI, London, ON, Canada
}

\section{A R T I C L E I N F O}

\section{Article history:}

Received 18 April 2011

Received in revised form 10 June 2011

Accepted 18 June 2011

\section{Keywords:}

Valproate

Anti-epileptic

Mood stabilizer

Lithium

Histone deacetylase (HDAC) inhibitor

Early growth factor receptor 1 (Egr1)

Mitogen-activated protein kinase (MAPK)

Extracellular signal-regulated kinase (ERK)

\begin{abstract}
A B S T R A C T
The mood stabilizer valproic acid (VPA) decreases neural progenitor proliferation and promotes neurogenesis in the adult hippocampus. However, the effects of VPA on progenitor cells in the adult subventricular zone (SVZ) are not as well characterized. Here we report VPA blocks neurosphere formation and inhibits DNA synthesis in cultured NSCs from the SVZ of adult mice. Inhibition of DNA synthesis is associated with the up-regulation of the differentiation transcription factors Egr1 and Neurod1 and down-regulation of transcription factors associated with "stemness". Co-treatment of VPA with the mood stabilizer lithium antagonizes the anti-proliferative effects of VPA on adult NSCs and abolishes VPA activation of Egr1. Co-treatment of VPA with the MEK1/2 inhibitor PD980589 similarly abolishes Egr1 activation consistent with VPA activation and lithium antagonism of MEK-ERK signaling in adult NSCs. However, Western blot reveals VPA significantly suppresses ERK2 phosphorylation in adult NSCs grown in proliferating culture conditions and that lithium co-treatment does not attenuate this effect. Combined the data indicate VPA inhibition of adult NSC proliferation and activation of Egr1 by VPA, along with the antagonism of these effects by lithium, are the effects of cumulative changes in multiple signaling pathways and are not attributable to a common kinase target.
\end{abstract}

Published by Elsevier Ireland Ltd.
Valproic acid (VPA, valproate) is a commonly prescribed anticonvulsant and mood stabilizer used in the treatment of epilepsy and bipolar disorder. VPA treatment reduces neuronal damage and aberrant neurogenesis associated with epileptic activity in adult rodents $[3,15]$. Conversely, VPA treatment promotes neurogenesis in non-pathological adult rodent models $[12,14]$ indicating the drug has complex, context-dependent effects. VPA affects various cell signaling systems that likely contribute to the drug's properties. These include mitogen activated protein kinase (MAPK) signaling $[12,16]$, glycogen synthase kinase-3 (GSK-3) activity [5] and inositol and phospholipid signaling [4,22]. Furthermore, at high doses VPA inhibits histone deacetylase (HDAC) activity [11] and HDACmediated responses are proposed to contribute to the differential neurogenic effects of VPA in the normal and epileptic adult rodent brain $[14,15,23]$.

Abbreviations: MEK, MAP kinase or ERK kinase; NaB, sodium butyrate; NSC, neural stem cell; VPA, valproic acid.

* Corresponding author at: Center for Neuroscience and Regenerative Medicine, Physiology and Genetics, Uniformed Services University of the Health Sciences, 4301 Jones Bridge Road, Bethesda, MD 20814, United States. Tel.: +1 301295 3206; fax: +1 3012951786 .

E-mail address: mdoughty@usuhs.mil (M.L. Doughty).
VPA-stimulated neurogenesis has been linked to activation of ERK pathway signaling in cultured embryonic forebrain progenitors and in the adult hippocampus and frontal cortex $[9,12,16]$. In this study, we have examined the effects of VPA on proliferating neural stem cells (NSCs) cultured from the SVZ of adult mice. We used neurosphere formation in culture as a model system to determine the molecular targets of VPA in proliferating adult NSCs.

8-10 week old male and female (equal numbers) C57BL/6 J mice were purchased from The Jackson Laboratory and housed in the Uniformed Services University's (USU) Center for Laboratory Animal Medicine. Animals were handled in accordance with procedures approved by the USU Institutional Animal Care and Use Committee (IACUC) and DoD regulations as published in DoD Directive 3216.1.

Adult neural stem cells (NSCs) were harvested and cultured from the subventricular zone (SVZ) of adult C57BL/6J mice as described in [25]. Cells were passaged a minimum 5 times prior to experimental analysis to ensure a high enrichment of multipotent stem cells.

Valproic acid sodium salt (Sigma) or sodium butyrate sodium salt (Sigma) was added to culture media to a final $1 \mathrm{mM}$ concentration at 3-h post-passage. For co-treatment experiments, lithium/selective kinase inhibitor was added $30 \mathrm{~min}$ prior to the addition of valproic acid/sodium butyrate. The final concentrations of co-treated drugs in media were: (1) $3 \mathrm{mM}$ lithium chloride 
(Sigma); (2) $30 \mu \mathrm{M}$ MEK1/2 inhibitor PD980589 (Cell Signaling Technology); (3) $10 \mu \mathrm{M}$ GSK-3 inhibitor SB 216763 (Sigma); and (4) $10 \mu \mathrm{M}$ CREB-CREB binding protein (CBP) interaction inhibitor 217505 (EMD Biosciences). An equal volume of DMSO/water only was added to the media of vehicle control cultures.

All procedures were performed according to the manufacturer's instructions (Invitrogen). For combined cell cycle analysis, adult NSCs cultures were pulsed overnight $(16 \mathrm{~h})$ with $10 \mu \mathrm{M} 5-$ ethynyl-2'-deoxyuridine (EdU) in cell culture media at $37^{\circ} \mathrm{C}, 5 \% \mathrm{CO}_{2}$ and co-labeled with CellCycle 488-red (7-AAD) and LIVE/DEAD ${ }^{\circledR}$ Fixable Violet stains. For combined drug treatments (VPA plus LiCl/PD980589/SB 216763/217505) adult NSC cultures were EdU pulsed for $4 \mathrm{~h}$ in cell culture media at $37^{\circ} \mathrm{C}, 5 \% \mathrm{CO}_{2}$. Flow cytometry and data collection were performed as described in [25].

Adult NSCs were incubated with a $0.2 \%$ solution of trypan-blue dye and small cell clusters/neurospheres excluding dye counted as described in [25].

Real-time quantitative reverse transcription PCR (qRT-PCR) was performed as described in [8]. Optimum primers were designed using NCBI's Primer BLAST. Primer sequence pairs used are: Actb GGCTGTATTCCCCTCCATCG and CCAGTTGGTAACAATGCCATGT; Egr1 GGGAGAGGCAGGAAAGACATTT and TCTGAGATCTTCCATCTGACCTAAGA; Gapdh CCCCVGCAAGGACACTGAGCAAGAG and GCCCCTCCTGTTATTATGGGGGTC; Hes1 TGGCGGCTTCCAAGTGGTGC and GATGACCGGGCCGCTGTGAG; Hprt1 ACGGGGGACATAAAAGTTATTGGTGG and ACCATTTTGGGGCTGTACTGCTTAAC; Hsp90 GCTTGCCGTGCGAGTCGGAC and CCACCTCCTCCTCTCCATGGTGC; c-Myc ACCCGCTCAACGACAGCAGC and CCGTGGGGAGGACTCGGAGG; Neurod1 CCGCCACACGCCTACA and CAAACTCGGCGGATGG; Sox2 GAAAGAAAGGAGAGAAGTTTGGAG and ATCTGGCGGAGAATAGTTGGG. Ct values were normalized to $\mathrm{Ct}$ values for Gapdh, Actb, Hprt1 and/or Hsp90. All values represent a minimum of 3 biological replicates.

Nuclear and cytoplasmic proteins were isolated from adult NSCs using NE-PER ${ }^{\circledR}$ reagents (Thermo Scientific) according to the manufacturer's instructions (Thermo Scientific) and Western blots of protein extracts (10-20 $\mu \mathrm{g}$ protein/lane) performed as described in [25]. Primary antisera/dilutions used were: 1:1000 rabbit monoclonal anti-CREB; 1:1000 rabbit monoclonal anti-phospho-CREB; $1: 1000$ rabbit monoclonal anti-GSK-3 $\beta ; 1: 1000$ rabbit polyclonal anti-phospho-GSK-3 $\alpha / \beta ; 1: 1000$ PathScan ${ }^{\circledR}$ Multiplex Western Cocktail I of rabbit polyclonals anti-phospho-p90-RSK, antiphospho-Akt, anti-phospho-p44/42 MAPK, anti-phopho-S6 and anti-EIF4e (all Cell Signaling Technology); 1000 mouse monoclonal anti-Gapdh (Millipore): or 1000 mouse monoclonal anti-Laminin A/C (BD Biosciences).

We first examined the effects of chronic VPA treatment on neurosphere formation by adult neural stem cells (NSCs). NSCs from the adult SVZ were grown in proliferating culture conditions with $1 \mathrm{mM}$ VPA for 2-7 days ( $1 \mathrm{mM}$ VPA has been shown to inhibit the proliferation of hippocampal progenitor cells and promote neurogenesis in culture, see $[14,23])$. VPA-treated adult NSCs produce fewer neurospheres of comparable size or density when compared to vehicle-treated controls (Fig. 1a). Reduced neurosphere formation is noticeable at $24 \mathrm{~h}$ treatment and persists for the full 7 days treatment regimen. Quantification at 7-days VPA treatment reveals an $8.1 \pm 3.0$ fold-decrease in the number of neurospheres (diameter $\geq 50 \mu \mathrm{m})$ in VPA treated cultures $(n=4)$. The number of small cell clusters ( $\geq 4$ cells, diameter $<50 \mu \mathrm{m}$ ) increases $5.0 \pm 2.1$ fold in VPA treated cultures $(n=4)$ consistent with the continued survival of non-neurosphere forming cells. Quantification of the toxic effects of $1 \mathrm{mM}$ VPA on adult NSCs using LIVE/DEAD staining and flow cytometry reveals VPA treatment results in a $9.6 \pm 4.2 \%$ reduction in cell viability compared to vehicle controls after $48 \mathrm{~h}$ treatment, a difference in cell viability that did not reach statistical significance
$(P=0.09$, unpaired $t$-test, $n=3)$. Combined these results indicate chronic VPA treatment results in a mildly toxic inhibition of adult NSC proliferation in culture.

We next quantified VPA's effects on DNA synthesis (S phase) by measuring incorporation rates of the thymidine analog nucleoside EdU (5-ethynyl-2'-deoxyuridine). Adult NSCs were treated with $1 \mathrm{mM}$ VPA or vehicle for $48 \mathrm{~h}$ in proliferating culture conditions and pulsed with $10 \mu \mathrm{M}$ EdU for the final $16 \mathrm{~h}$ of treatment. Flow cytometry reveals EdU incorporation is 3-fold lower in live cell gated adult mouse NSCs treated with VPA compared to vehicle controls $(18.3 \pm 2.1 \%$ versus vehicle $54.5 \pm 7.5 \%$ vehicle, $P<0.001$, see Fig. $1 \mathrm{~b}$ and c). To determine whether VPA suppression of S phase DNA synthesis is associated with cell cycle arrest, we estimated cell cycle stage by flow cytometry using the Dean-Jett-Fox model (Flowjo software). This revealed a small, statistically insignificant increase in the percentage of cells in $G 1$ phase $(51.1 \pm 1.4 \%$ versus $47.9 \pm 1.3 \%$ vehicle, $P>0.05)$ and corresponding decrease in the percentage of cells in G2/M phase $(19.0 \pm 1.3 \%$ versus $24.8 \pm 1.7 \%$ vehicle, $P>0.05)$ of the cell cycle (Fig. 1c). Accordingly, we conclude chronic VPA treatment inhibits adult NSC proliferation in culture by $S$ phase inhibition unassociated with significant arrest in either G1 or G2/M phase of the cell cycle.

Lithium, like VPA, is a commonly prescribed mood stabilizer [10] and the two drugs have overlapping mechanisms of activity $[5,9,17,22]$. We examined the effects of lithium co-treatment on the anti-proliferative effects of VPA to determine common molecular targets. Adult NSCs grown in proliferating conditions were treated for $48 \mathrm{~h}$ with VPA, VPA plus lithium chloride ( $3 \mathrm{mM} \mathrm{LiCl}$ ) or vehicle, pulsed with EdU for the final $4 \mathrm{~h}$ of treatment and the cells analyzed by flow cytometry. Co-treatment of VPA with lithium results in significantly higher adult NSC proliferation rates than those treated with VPA alone (Fig. 1d). The addition of lithium to VPA increases EdU incorporation rates in adult NSCs 1.8-fold (VPA $9.59 \pm 1.07$ versus VPA and lithium $17.23 \pm 1.71$ EdU+ve, $P<0.01$, $n=4$ ), indicating an antagonistic response to the addition of lithium.

To assess the importance of HDAC inhibitor-mediated effects in the antagonism of VPA proliferative responses by lithium, we examined the effects of lithium co-treatment with sodium butyrate (NaB), a molecule with similar class I/II HDAC inhibitor properties to VPA. Using the same culture assay, $1 \mathrm{mM} \mathrm{NaB}$ inhibits neurosphere formation by NSCs from the adult mouse SVZ [25]. In contrast to VPA/lithium co-treatment, $\mathrm{NaB} /$ lithium co-treatment does not significantly decrease the anti-proliferative effects of $\mathrm{NaB}(P=0.63$, $n=4$ ), see Fig. 2 . Combined the data suggests lithium antagonizes VPA sensitive cell signaling mechanisms other than HDAC inhibition to reduce the anti-proliferative effects of VPA on adult NSCs.

We measured mRNA levels in adult NSCs treated with VPA or vehicle to identify changes in adult NSC gene expression associated with treatment effects. Real-time PCR reveals chronic VPA treatment up-regulates the immediate early gene transcription factor Egr1 (Krox24, Zif268) and more robustly the pro-neural transcription factor Neurod1 in adult NSCs (Fig. 2a). In contrast, Hes1, c-Myc and Sox 2 transcription factors associated with the maintenance of a stem/progenitor cell state are down-regulated in adult NSCs (Fig. 2a).

We next asked if these VPA-modulated changes in transcription correlated with the antagonism of VPA effects by lithium co-treatment. Real-time PCR revealed co-treatment of VPA with lithium reverses VPA-mediated increases in Egr1 transcription (Fig. 2b). In contrast, Neurod1 up-regulation and Hes1, c-Myc and Sox2 down-regulation by VPA were unaffected by lithium cotreatment (not shown). This suggests a specific correlation between Egr1 activation and the antagonism of VPA inhibition of proliferation by lithium.

Egr1 is induced by growth factors through ERK/CREB (CAMPresponse element-binding protein) activation (for review see [2]) 
a

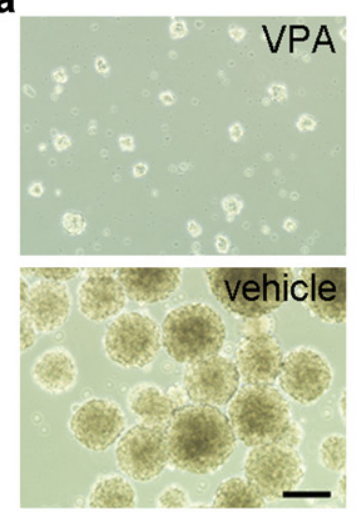

b

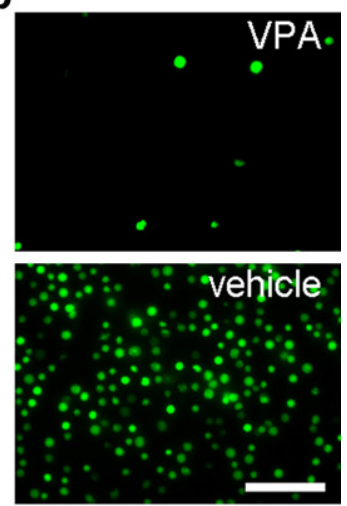

C

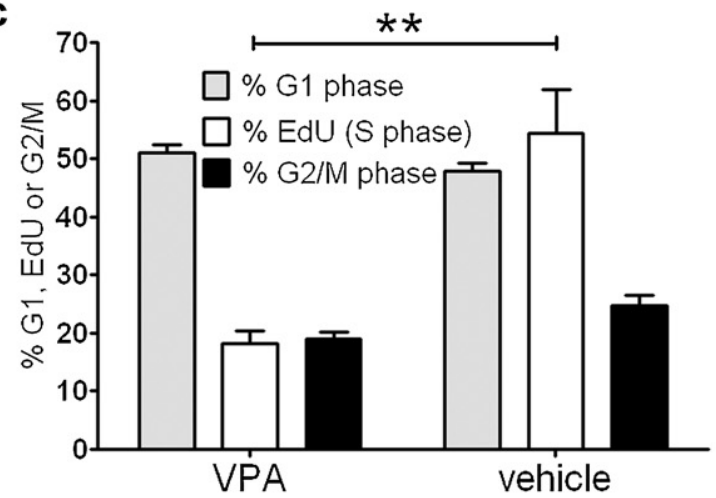

d

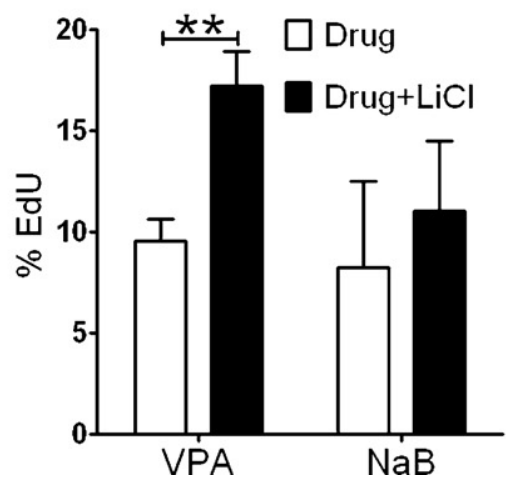

Fig. 1. Chronic VPA treatment inhibits adult mouse NSC proliferation and neurosphere formation in culture. (a) Light micrographs of adult NSC cultures following chronic 7-day treatment with $1 \mathrm{mM}$ VPA or vehicle. Adult NSCs were grown in proliferating culture conditions and treated with VPA or water vehicle. Scale bar $=100 \mu \mathrm{m}$. (b) Fluorescence micrographs of $1 \mathrm{mM}$ VPA and vehicle treated adult NSCs pulse-labeled with EdU for flow cytometry. Scale bar $=50 \mu \mathrm{m}$. (c) Analysis of EdU incorporation and DNA content by flow cytometry reveals chronic $1 \mathrm{mM}$ VPA treatment significantly inhibits DNA synthesis $\left({ }^{* *} P<0.01\right)$ by adult NSCs in culture but is not associated with significant arrest in G1 or G2/M phase $(P$-values $>0.05)$ of the cell cycle (1-way ANOVA with post hoc Newman-Keuls multiple comparison tests, $n=3$ ). Adult NSCs were treated with $1 \mathrm{mM}$ VPA or vehicle for $48 \mathrm{~h}$ in proliferating culture conditions, pulsed with EdU for the final $16 \mathrm{~h}$ of treatment and processed for flow cytometry. (d) Co-treatment with lithium significantly reduces the anti-proliferative effects of VPA but not of the HDAC inhibitor sodium butyrate (NaB). Adult NSCs were treated with $1 \mathrm{mM}$ VPA, $1 \mathrm{mM}$ VPA plus $3 \mathrm{mM}$ lithium chloride $(\mathrm{LiCl}), 1 \mathrm{mM} \mathrm{NaB}$ or $1 \mathrm{mM} \mathrm{NaB}$ plus $3 \mathrm{mM} \mathrm{LiCl}$ for $48 \mathrm{~h}$, pulsed with EdU for the final $4 \mathrm{~h}$ of treatment and processed for flow cytometry. EdU incorporation is significantly increased 1.8 fold by lithium co-treatment with VPA compared to VPA alone $\left({ }^{* *} P<0.01\right.$, $t$-test, $\left.n=4\right)$. In contrast, lithium co-treatment with $\mathrm{NaB}$ results in a smaller 1.3 fold increase in EdU incorporation compared to $\mathrm{NaB}$ alone that is not significantly different $(P=0.63, t$-test, $n=4)$. a

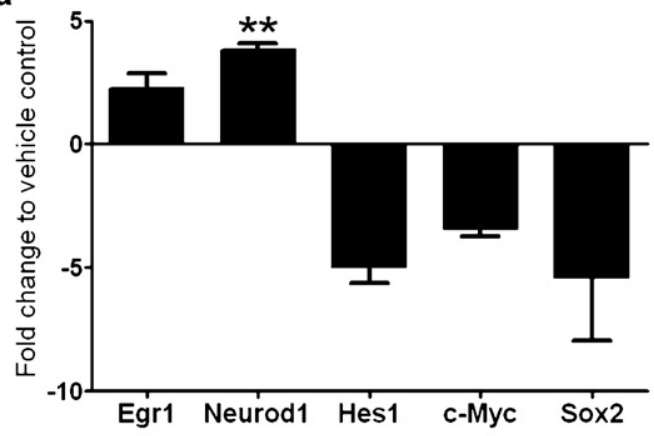

b

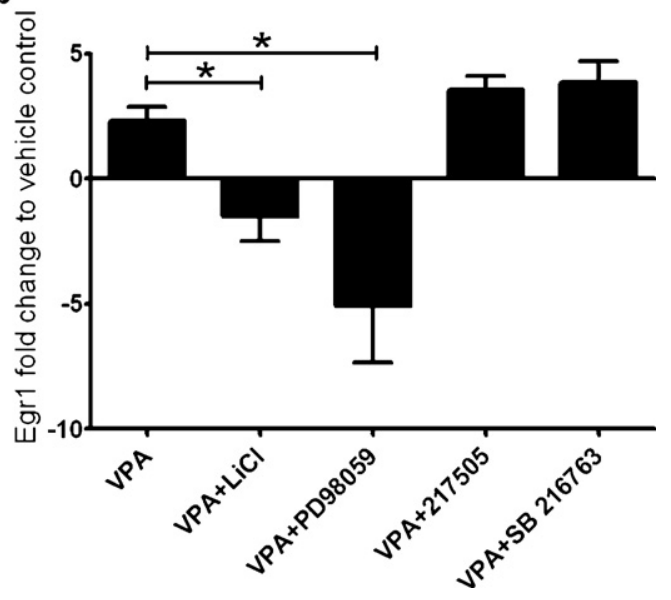

Fig. 2. Chronic VPA treatment activates Egr1 gene transcription in proliferating adult NSCs via a lithium and MEK1/2 sensitive mechanism. Adult NSCs were treated with $1 \mathrm{mM}$ VPA or vehicle for $48 \mathrm{~h}$ in proliferating culture conditions and gene expression measured by qRT-PCR. For co-treatments, lithium/selective kinase inhibitor was added 30 min prior to the addition of VPA. Cells were harvested for qRT-PCR after $48 \mathrm{~h}$ treatment and all qPCR values calculated from $\geq 3$ biological replicates. (a) Chronic VPA treatment up-regulates differentiation transcription factors Egr1 and more robustly Neurod1 in adult NSCs grown in proliferating culture conditions $\left({ }^{* *} P<0.01, t\right.$-test, $n=5$ ). In contrast, Hes 1 , c-Myc and Sox 2 transcription factors associated with the maintenance of a stem/progenitor cell state are down-regulated. (b) Co-treatment with lithium or with the MEK1/2 inhibitor PD98095 blocks VPA activation of Egr1 transcription in adult NSCs grown in proliferating culture conditions. Adult NSCs were treated with $3 \mathrm{mM} \mathrm{LiCl}, 30 \mu \mathrm{M}$ of the MEK1/2 inhibitor PD98095, $10 \mu \mathrm{M}$ CREB-CBP interaction inhibitor 217505, $10 \mu \mathrm{M}$ of the GSK-3 inhibitor SB 216763 or vehicle $30 \mathrm{~min}$ prior to the addition of $1 \mathrm{mM}$ VPA. VPA-mediated increase in Egr1 transcript levels is significantly suppressed by co-treatment with $\mathrm{LiCl}$ or PD98095 $\left({ }^{*} P<0.051\right.$-way ANOVA with post hoc Newman-Keuls multiple comparison tests, $n \geq 3$ ).

and we speculated antagonism of VPA effects on ERK/CREB signaling by lithium could account for changes in Egr1 transcription. To test this hypothesis we measured mRNA levels of Egr1 in adult NSCs treated with VPA plus selective kinase inhibitors. We co-treated cells with PD98059 (30 $\mu \mathrm{M})$, a highly selective inhibitor of MEK1/2 activation and the MEK-ERK cascade [7], with $217505(10 \mu \mathrm{M})$ a small molecule shown to disrupt the interaction between CREB and CREB binding protein (CBP) [1] and, in view of the known inhibitory effects of lithium on GSK-3 activity [24], with the GSK3 inhibitor SB $216763(10 \mu \mathrm{M})$. This analysis revealed inhibition of MEK1/2 but not CREB-CBP interaction or GSK-3 activity antagonized VPA-mediated increases in Egr1 transcription (Fig. 2b). This indicates VPA modulates MEK-ERK signaling independent of the downstream transcriptional regulator CREB or GSK-3 to activate Egr1.

Down-regulation of Egr1 with MEK-ERK inhibition or lithium raises the possibility lithium co-treatment inhibits MEK-ERK signaling to antagonize the effects of VPA. Indeed, VPA has been shown to activate ERK signaling in embryonic cortical progenitor 
a

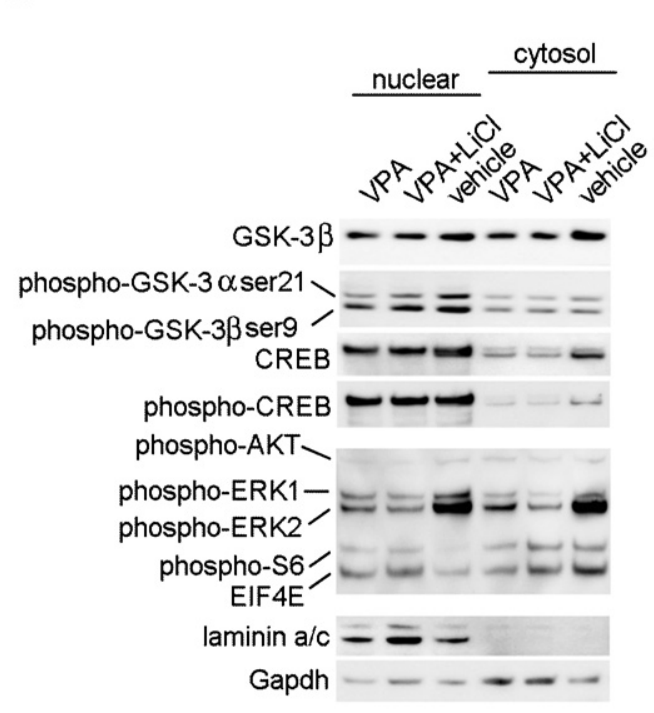

b

b Nuclear

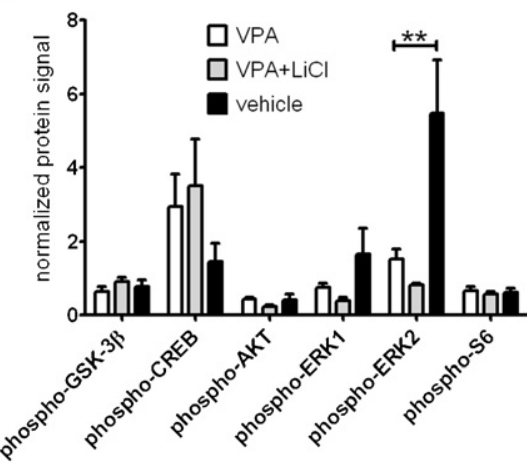

Cytosol

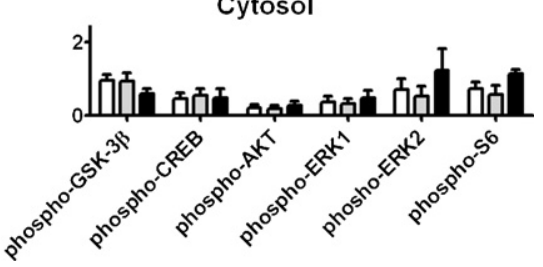

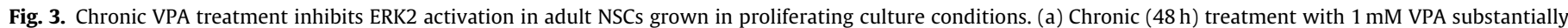

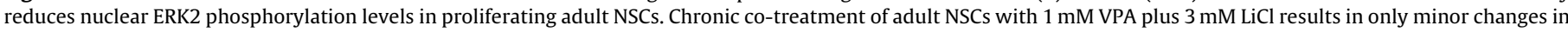

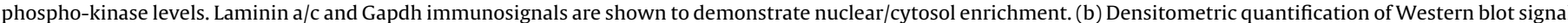

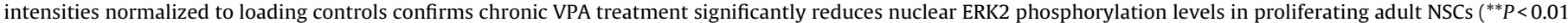

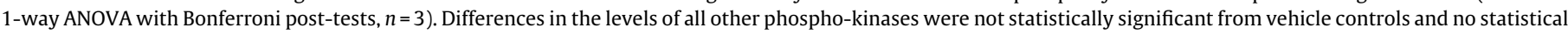

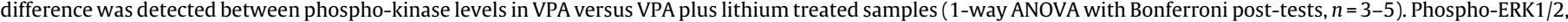

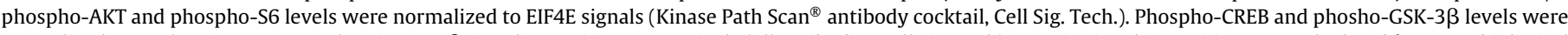

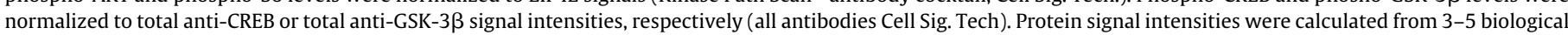
replicates.

cells $[12,16]$. We analyzed the phosphorylation levels of a selection of kinases in adult NSCs to identify VPA sensitive cell signaling mechanisms antagonized by lithium. Western blot reveals chronic VPA treatment significantly reduces rather than enhance ERK2 phosphorylation in adult NSCs grown in proliferating culture conditions $(P<0.01)$ (Fig. 3). Furthermore, lithium co-treatment does not attenuate the inhibitory effect of VPA on ERK2 phosphorylation (Fig. 3); each kinase shows only small, non-significant changes to their phosphorylation in response to lithium when compared to VPA alone $(P>0.05, n \geq 3)$. Taken together the Western blot data suggest the reduction of VPA inhibition of adult NSC proliferation by lithium co-treatment is the effect of cumulative changes in multiple signaling pathways rather than via a common kinase target.

In this study we demonstrate the mood stabilizer valproic acid (VPA) modulates gene expression and kinase activity in adult NSCs to inhibit proliferation and neurosphere formation in culture. VPA inhibition of adult NSC proliferation was associated with the transcriptional activation of the immediate early gene transcription factor Egr1, a gene linked to de novo neurogenesis functions in adult mice $[20,21]$. Egr1 is activated by nerve growth factor (NGF) stimulation of the ERK pathway in PC12 cells and is required for NGF-induced neurite outgrowth $[13,18]$ and VPA has been shown to activate ERK signaling in the developing embryonic cortex [16]. However, chronic VPA treatment suppressed ERK2 phosphorylation in adult NSCs. Furthermore, lithium antagonism of the anti-proliferative effects and activation of Egr1 by VPA was not associated with lithium induced changes in ERK2 phosphorylation. Combined these data support the idea that VPA suppresses proliferation and activates Egr1 differentiation signals in cultured adult NSCs via the modulation of multiple signaling pathways.

There is good evidence to suggest reduced ERK2 phosphorylation and suppressed MAPK-ERK signaling accounts for the inhibition of adult NSC proliferation by VPA. FGF-2 is a potent inducer of adult NSC proliferation, inducing sustained ERK1/2 phos- phorylation in adult NSCs, and genetic studies confirm MAPK-ERK signaling is required for the maintenance of adult NSC proliferation and suppression of differentiation by FGF-2 [19]. Interestingly, genetic attenuation of MAPK-ERK signaling activates Neurod1 expression and promotes differentiation in FGF-2 treated adult NSCs [19]. However, VPA activation of Neurod1 in adult hippocampal progenitors, like Egr1 activation in our adult SVZ NSCs, is independent of MAPK-ERK pathway activation [14]. In the case of Neurod1, transcriptional activation of pro-neural basic helix-loophelix transcription factors upstream of Neurod1 [23] could account for Neurod1 up-regulation. Chromatin immunoprecipitation data from our laboratory (unpublished) indicates VPA indirectly activates Egr1. It is possible Egr1 activation involves multiple molecular mechanisms, as appears to be the case for VPA induction of Bcl-2 expression in human neuroblastoma cells in which VPA triggers a signaling cascade of ERK and/or PI3K (phosphatidylinositol 3kinase) activation of RSK (p90 ribosomal S6 kinase) to up-regulate $\mathrm{Bcl}-2$ transcription, and yet chemical inhibition of ERK/PI3K or RSK shRNA knockdown attenuates but does not abolish Bcl-2 induction by VPA [6]. In summary, our data indicate the effects of VPA alone or in co-treatment with lithium on cultured adult NSCs are the result of cumulative changes in multiple signaling pathways and are not attributable to a common kinase target.

\section{Acknowledgment}

This work is supported by a grant from the Center for Neuroscience and Regenerative Medicine (CNRM).

\section{References}

[1] J.L. Best, C.A. Amezcua, B. Mayr, L. Flechner, C.M. Murawsky, B. Emerson, T. Zor, K.H. Gardner, M. Montminy, Identification of small-molecule antagonists that 
inhibit an activator: coactivator interaction, Proc. Natl. Acad. Sci. U.S.A. 101 (2004) 17622-17630.

[2] B. Bozon, A. Kelly, S.A. Josselyn, A.J. Silva, S. Davis, S. Laroche, MAPK, CREB and zif268 are all required for the consolidation of recognition memory, Philos. Trans. R. Soc. Lond. B: Biol. Sci. 358 (2003) 805-814.

[3] C. Brandt, A.M. Gastens, M. Sun, M. Hausknecht, W. Loscher, Treatment with valproate after status epilepticus: effect on neuronal damage, epileptogenesis, and behavioral alterations in rats, Neuropharmacology 51 (2006) 789-804.

[4] M.C Chang, M.A. Contreras, T.A. Rosenberger, J.J. Rintala, J.M. Bell, S.I. Rapoport, Chronic valproate treatment decreases the in vivo turnover of arachidonic acid in brain phospholipids: a possible common effect of mood stabilizers, J. Neurochem. 77 (2001) 796-803.

[5] G. Chen, L.D. Huang, Y.M. Jiang, H.K. Manji, The mood-stabilizing agent valproate inhibits the activity of glycogen synthase kinase-3, J. Neurochem. 72 (1999) 1327-1330

[6] T.K. Creson, P. Yuan, H.K. Manji, G. Chen, Evidence for involvement of ERK, PI3K, and RSK in induction of Bcl-2 by valproate, J. Mol. Neurosci. 37 (2009) 123-134.

[7] C.M Crews, A. Alessandrini, R.L. Erikson, The primary structure of MEK, a protein kinase that phosphorylates the ERK gene product, Science 258 (1992) 478-480.

[8] C.L. Dalgard, Q. Zhou, T.G. Lundell, M.L. Doughty, Altered gene expression in the emerging cerebellar primordium of Neurog1(-/-) mice, Brain Res. (2011).

[9] H. Einat, P. Yuan, T.D. Gould, J. Li, J. Du, L. Zhang, H.K. Manji, G. Chen, The role of the extracellular signal-regulated kinase signaling pathway in mood modulation, J. Neurosci. 23 (2003) 7311-7320.

[10] S.D. Friedman, S.R. Dager, A. Parow, F. Hirashima, C. Demopulos, A.L. Stoll, I.K. Lyoo, D.L. Dunner, P.F. Renshaw, Lithium and valproic acid treatment effects on brain chemistry in bipolar disorder, Biol. Psychiatry 56 (2004) 340-348.

[11] D.R. Grayson, M. Kundakovic, R.P. Sharma, Is there a future for histone deacetylase inhibitors in the pharmacotherapy of psychiatric disorders? Mol. Pharmacol. 77 (2010) 126-135.

[12] Y. Hao, T. Creson, L. Zhang. P. Li, F. Du, P. Yuan, T.D. Gould, H.K. Manji, G. Chen, Mood stabilizer valproate promotes ERK pathway-dependent cortical neuronal growth and neurogenesis, J. Neurosci. 24 (2004) 6590-6600.

[13] T. Harada, T. Morooka, S. Ogawa, E. Nishida, ERK induces p35, a neuron-specific activator of Cdk5, through induction of Egr1, Nat. Cell Biol. 3 (2001) 453459 .
[14] J. Hsieh, K. Nakashima, T. Kuwabara, E. Mejia, F.H. Gage, Histone deacetylase inhibition-mediated neuronal differentiation of multipotent adult neural progenitor cells, Proc. Natl. Acad. Sci. U.S.A. 101 (2004) 16659-16660.

[15] S. Jessberger, K. Nakashima, G.D. Clemenson Jr., E. Mejia, E. Mathews, K. Ure, S. Ogawa, C.M. Sinton, F.H. Gage, J. Hsieh, Epigenetic modulation of seizureinduced neurogenesis and cognitive decline, J. Neurosci. 27 (2007) 5967-5970.

[16] G.A. Jung J.Y. Yoon, B.S. Moon, D.H. Yang H.Y. Kim, S.H. Lee, V. Bryja, E. Arenas, K.Y. Choi, Valproic acid induces differentiation and inhibition of proliferation in neural progenitor cells via the beta-catenin-Ras-ERK-p21Cip/WAF1 pathway, BMC Cell Biol. 9 (2008) 66.

[17] Y. Leng, M.H. Liang, M. Ren, Z. Marinova, P. Leeds, D.M. Chuang, Synergistic neuroprotective effects of lithium and valproic acid or other histone deacetylase inhibitors in neurons: roles of glycogen synthase kinase-3 inhibition, J. Neurosci. 28 (2008) 2576-2580.

[18] Y. Levkovitz, K.J. O’Donovan, J.M. Baraban, Blockade of NGF-induced neurite outgrowth by a dominant-negative inhibitor of the egr family of transcription regulatory factors, J. Neurosci. 21 (2001) 45-52.

[19] D.K. Ma, K. Ponnusamy, M.R. Song G.L. Ming H. Song, Molecular genetic analysis of FGFR1 signalling reveals distinct roles of MAPK and PLCgamma1 activation for self-renewal of adult neural stem cells, Mol. Brain 2 (2009) 16.

[20] N. Mandairon, J. Sacquet, S. Garcia, N. Ravel, F. Jourdan, A. Didier, Neurogenic correlates of an olfactory discrimination task in the adult olfactory bulb, Eur. J. Neurosci. 24 (2006) 3578-3580.

[21] A. Tashiro, H. Makino, F.H. Gage, Experience-specific functional modification of the dentate gyrus through adult neurogenesis: a critical period during an immature stage, J. Neurosci. 27 (2007) 3252-3260.

[22] R.S. Williams, L. Cheng, A.W. Mudge, A.J. Harwood, A common mechanism of action for three mood-stabilizing drugs, Nature 417 (2002) 292-295.

[23] I.T. Yu, J.Y. Park, S.H. Kim, J.S. Lee, Y.S. Kim, H. Son, Valproic acid promotes neuronal differentiation by induction of proneural factors in association with H4 acetylation, Neuropharmacology 56 (2009) 473-480.

[24] F. Zhang, C.J. Phiel, L. Spece, N. Gurvich, P.S. Klein, Inhibitory phosphorylation of glycogen synthase kinase-3 (GSK-3) in response to lithium. Evidence for autoregulation of GSK-3, J. Biol. Chem. 278 (2003) 33067-33070.

[25] Q. Zhou, C.L. Dalgard, C. Wynder, M.L. Doughty, Histone deacetylase inhibitors SAHA and sodium butyrate block G1-to-S cell cycle progression in neurosphere formation by adult subventricular cells, BMC Neurosci. 12 (2011) 50. 